\title{
In Vivo Tracking of Chemokine Receptor CXCR4-Engineered Mesenchymal Stem Cell Migration by Optical Molecular Imaging
}

\author{
Senthilkumar Kalimuthu, Ji Min Oh, Prakash Gangadaran, Liya Zhu, Ho Won Lee, \\ Ramya Lakshmi Rajendran, Se hwan Baek, Yong Hyun Jeon, Shin Young Jeong, \\ Sang-Woo Lee, Jaetae Lee, and Byeong-Cheol Ahn
}

Department of Nuclear Medicine, Kyungpook National University School of Medicine and Hospital, Daegu, Republic of Korea

Correspondence should be addressed to Byeong-Cheol Ahn; abc2000@knu.ac.kr

Received 15 February 2017; Revised 4 May 2017; Accepted 11 May 2017; Published 27 June 2017

Academic Editor: Renke Li

Copyright (c) 2017 Senthilkumar Kalimuthu et al. This is an open access article distributed under the Creative Commons Attribution License, which permits unrestricted use, distribution, and reproduction in any medium, provided the original work is properly cited.

\begin{abstract}
CXCR4, the stromal cell-derived factor-1 receptor, plays an important role in the migration of hematopoietic progenitor/stem cells to injured and inflamed areas. Noninvasive cell tracking methods could be useful for monitoring cell fate. Therefore, in this study, we evaluated the efficacy of an intravenous infusion of genetically engineered mesenchymal stem cells (MSCs) overexpressing CXC chemokine receptor 4 (CXCR4) to home to the tumor, by optical imaging. We constructed a retroviral vector containing CXCR with dual reporter genes, eGFP and Fluc2, under the control of an EF1 $\alpha$ promoter (pBABE-EF1 $\alpha$-CXCR4-eGFP-IRES-Fluc2). We also developed an eGFP-Fluc2 construct in the Retro-X retroviral vector (Retro-X-eGFP-Fluc2). MSCs were transduced with retroviruses to generate CXCR4-overexpressing MSCs (MSC-CXCR4/Fluc2) and MSCs (MSC/Fluc2). CXCR4 mRNA and protein expression was confirmed by RT-PCR and Western blotting, respectively, and it was higher in MSC-CXCR4/Fluc2 than in naive MSCs. eGFP expression was confirmed by confocal microscopy. The transfected MSC-CXCR4/Fluc2 cells showed higher migratory capacity than naive MSCs observed in Transwell migration assay. The in vivo migration of CXCR4-overexpressing MSCs to MDAMB231/Rluc tumor model by BLI imaging was also confirmed. Intravenous delivery of genetically modified MSCs overexpressing CXCR4 with a Fluc2 reporter gene may be a useful, noninvasive BLI imaging tool for tracking cell fate.
\end{abstract}

\section{Introduction}

Worldwide, there are 1.38 million women with breast cancer [1]. Breast cancer is one of the most common cancers among women, especially in less developed countries and the second leading cause of cancer death [2]. Most patients with breast cancer eventually undergo chemotherapy. However, only a few patients treated with chemotherapy show longterm reduction, and response failures are common due to chemoresistant tumors [3]. Triple negative breast cancer (TNBC) is a subtype of tumors that do not clinically express human epidermal growth factor receptor 2 (HER-2), progesterone receptor (PR), or estrogen receptor (ER). This subtype, which is associated with poor prognosis, accounts for approximately $15-20 \%$ breast cancers. However, TNBC is accountable for a disparate number of deaths, and there is no effective, specific-targeted therapy available [4]. Mesenchymal stem cell- (MSC-) based homing studies may lead to a better approach for treating such breast cancers.

MSCs are self-renewing, multipotent, progenitor cells with multilineage potential that can differentiate into cells of mesodermal origin, such as adipocytes, osteocytes, and chondrocytes $[5,6]$. MSC-mediated gene therapy has been investigated as an option for the treatment of various diseases, including articular cartilage damage, hemophilia, and myocardial infarction [7-11]. MSCs are most commonly isolated from bone marrow [12], although they are also isolated from other tissues, including adipose tissue $[13,14]$, 
the placenta [15], amniotic fluid [16], and umbilical cord blood $[17,18]$. Owing to their accessibility and convenient expansion protocols, MSCs have been recognized as promising candidates for cellular therapy. Bone marrow is a rich source of MSCs; therefore, it is often used for isolating MSCs. MSCs can be expanded by ex vivo culture, thereby making them good vehicles for in vivo gene delivery.

MSCs have the ability to home to damaged tissue sites. When MSCs are systemically delivered or exogenously administered to humans and animals, they specifically migrate to inflammation sites [5], although many intravenously administered MSCs become trapped in the lungs $[19,20]$. MSC homing to inflammation sites has been demonstrated, and numerous cell trafficking-related molecules are involved, mainly adhesion molecules, chemokines, and matrix metalloproteinases. Among these, the CXC motif chemokine ligand 12-CXC motif chemokine receptor 4 (CXCR4) and CC motif chemokine ligand 2-CC motif chemokine receptor 2 (CCR2) ligand-receptor pairs have been actively studied $[21,22]$.

Although CXCR4 is highly expressed by MSCs within the bone marrow [23], its expression is markedly reduced during ex vivo expansion [24], which decreases their ability to home to injured sites. Therefore, the activation of CXCR4 could make MSCs migrate to CXCR4 ligands. CXCR4, the stromal cell-derived factor-1 (SDF) receptor, plays an important role in the migration of hematopoietic progenitor/stem cells [25]. The overexpression of CXCR4 in human hematopoietic stem cells or CD34+ progenitors has been shown to improve chemotaxis, migration, and homing [26]. However, there are limited noninvasive studies showing the migration potency of these therapeutic CXCR4-overexpressing cells to cancer cells in animal models.

Recent developments in noninvasive imaging tools continue to reinforce the utility of molecular imaging for biological research. These imaging technologies, coupled with the development of cell-based therapies, are leading a transformation in cell tracking. The transplanted cells are visualized via molecular imaging tools, which shows the fate, function, migration, and homing of the cells, and such in vivo cell monitoring strategies are highly valuable for the development of cell-based therapies [27]. Moreover, cell tracking using bioluminescence imaging (BLI) offers the highest sensitivity in small animal experiments due to the absence of endogenous luciferase expression in mammalian cells [28]. Therefore, in the current study, we established CXCR4-overexpressing MSCs, containing a Fluc2 reporter gene, and evaluated their in vivo migrating efficiency to triple negative breast cancer by BLI.

\section{Materials and Methods}

2.1. Chemicals. DMEM-high was obtained from Hyclone (Logan, UT, USA). Antibiotics were obtained from GibcoInvitrogen (Carlsbad, CA, USA), and the anti-CXCR4 antibody (rabbit polyclonal) was obtained from Abcam (Cambridge, MA, USA). The CXCR4-eGFP vector was a kind gift from Dr. Peter L. Hordijk (University of Amsterdam,
Netherlands). The pBABE vector was purchased from Addgene (Cambridge, MA, USA).

2.2. Cell Culture. Bone marrow-derived mouse MSCs were purchased from GIBCO (Invitrogen). Cells were grown in DMEM-high with $10 \%$ fetal bovine serum and $1 \%$ antibiotic-antimycotic (Gibco, Invitrogen) and maintained in a humidified incubator at $37^{\circ} \mathrm{C}$ with $5 \% \mathrm{CO}_{2}$.

2.3. Retroviral Vector and MSC Transduction. To track transplanted cells in vivo, MSCs were transduced with the retroviral vector pBABE carrying an EF1 $\alpha$ promoter for CXCR4 expression. To generate a triple gene construct, we inserted the CXCR4-eGFP cassette along with Fluc2 by using linker molecules and internal ribosome entry sites (IRES). The designed construct was inserted into the $\mathrm{pBABE}$ vector after under the control of the $\mathrm{EF} 1 \alpha$ promoter to generate the final construct, pBABE-EF1 $\alpha$-CXCR4-eGFP-IRES-Fluc2. We also generated another construct, Retro-X-eGFP-IRESFluc2 without CXCR4. The gene construct was shown in Additional File 1 available online at https://doi.org/10.1155/ 2017/8085637. Both plasmid retroviruses were produced in Gryphon E (Allele Biotechnology, San Diego, CA, USA) cells after transfection using the $\mathrm{CaPO}_{4}$ method. After 2 days, the medium was collected and filtered, and the filtrate was then transduced into MSCs. The transduced MSCs, with and without CXCR4 overexpression, were sorted to generate stable cell lines and called MSC-CXCR4/Fluc2 and MSC/Fluc2, respectively. The Fluc activity was measured by IVIS Lumina II by adding D-Luciferin $150 \mu \mathrm{g} / 100 \mu \mathrm{l}$ final concentration.

2.4. Confocal Microscopy. Naive MSCs, MSC/Fluc2, and MSC-CXCR4/Fluc2 cells $\left(5 \times 10^{5}\right)$ were plated in an 8 -well cell chamber. Twenty-four hours after plating, the cells were washed twice with PBS, fixed with $4 \%$ paraformaldehyde, and washed three times with PBS. Finally, the slides were mounted with a coverslip using Vecta shield mounting medium containing DAPI (Vector Laboratories, Burlingame, CA, USA), and eGFP images were visualized by confocal laser microscopy (LSM 5 exciter; Zeiss, Germany).

2.5. Flow Cytometry. To confirm the identity of CXCR4transduced MSCs, we assessed eGFP expression by flow cytometry. After stable transduction of MSC-CXCR4/Fluc2 cells, we compared the eGFP expression of MSC-CXCR4/ Fluc2 cells to that of naive MSCs.

2.6. Lentiviral Transduction of Breast Cancer Cells. Triple negative breast cancer cells (MDAMB231) were transduced with lentiviral particles expressing mCherry-Rluc under the control of the CMV promoter (GeneCopoeia, Rockville, MD, USA). After transfection, cells were selected with puromycin to generate a stable cell line. The stable cell line was named MDAMB231/Rluc, and the cells were screened by optical imaging and fluorescence microscopy. For Rluc activity, substrate coelenterazine h (CTZ) (Perkin Elmer, Waltham, MA, USA) was added to each well, and Rluc activity was determined by BLI (Bioluminescence imaging). 
2.7. RT-PCR. The transduced MSC-CXCR4/Fluc2 cell line was screened by RT-PCR. Total RNA was isolated from naive and transduced MSCs with TRIzol reagent (Invitrogen). The concentration and purity of the total RNA was determined by NanoDrop 2000 (Thermo Scientific, Wilmington, DE, USA), the ratio of which was 1.6-2.0. cDNA was synthesized from $1 \mu \mathrm{g}$ of total RNA using the TOPscript ${ }^{\mathrm{TM}} \mathrm{cDNA}$ synthesis kit (Enzynomics, Daejeon, South Korea) according to the manufacturer's protocol. For CXCR4 gene amplification, glyceraldehyde-3-phosphate dehydrogenase $(G A P D H)$ was used as a control for reverse transcription. The PCR reaction was performed with the i-Taq TM DNA Polymerase kit (iNtRON Biotechnology, Westchester, NY, USA) in an Eppendorf master cycler. The amplification protocol included an initial denaturation step at $95^{\circ} \mathrm{C}$ for $7 \mathrm{~min}$ and followed by 35 cycles of denaturation at $94^{\circ} \mathrm{C}$ for $40 \mathrm{~s}$ and extension at $60^{\circ} \mathrm{C}$ for $30 \mathrm{~s}$, with a final extension at $72^{\circ} \mathrm{C}$ for $10 \mathrm{~min}$. Amplification products were analyzed electrophoretically on a $1.0 \%$ agarose gel containing $0.5 \mathrm{mg}$ of ethidium bromide (Bioneer, Alameda, CA, USA). The PCR bands were visualized with a Biodoc IT imaging system (UVP, Upland, CA, USA).

2.8. Immunoblot Analysis. Cell lysates containing equal amounts of protein $(40 \mu \mathrm{g})$ were separated by SDS-PAGE and electrophoretically transferred to a PVDF membrane. The PVDF membrane was blocked with 5\% nonfat dry milk in PBS-Tween-20 $(0.1 \%, v / w)$ for 2-4 hours. The membrane was incubated with an anti-CXCR4 antibody (rabbit polyclonal, ab2074-Abcam, 1:1000) overnight. Then, an HRPconjugated anti-rabbit (Cell Signaling, Beverly, MA, USA) was used as the secondary antibody. Immunoreactive protein was visualized by chemiluminescence (Power OptiECL, Animal Genetics, South Korea) and quantified with Image Scanner. $\beta$-actin was used as an internal control.

2.9. Transwell Migration Assay. Migration assays were carried out in a 24-well Transwell using polycarbonate membranes with $8 \mu \mathrm{m}$ pores (Corning Costar, Cambridge, MA, USA). MSC-CXCR4/Fluc2, at a density of $1 \times 10^{5}$ cells in $500 \mu \mathrm{l}$ of serum-free medium, were placed in the upper chamber of the Transwell assembly. The lower chamber contained $0.5 \%$ FBS. After incubation at $37^{\circ} \mathrm{C}$ and $5 \% \mathrm{CO}_{2}$ for $6 \mathrm{~h}$, the upper surface of the membrane was scraped gently to remove nonmigrating cells and washed with PBS. Then, the migrated cells on the lower surface of the membrane were fixed in $4 \%$ paraformaldehyde for 15 minutes and stained with $0.1 \%$ crystal violet for 10 minutes. The number of migrating cells was photographed under a phase contrast microscope, and migrated cells were counted three different fields.

2.10. In Vivo MSC Migration to Tumors. BLI was used to assess the in vivo migration of transduced MSCs. All animal experiments were performed in accordance with institutional guidelines and were approved by the Institutional Animal Care and Use Committee of the Kyungpook National University of Korea. MDAMB231/Rluc breast cancer tumor xenografts were established in 6-week-old female nude mice by injection of $5 \times 10^{6}$ cells into the right flank. Tumor production 1 month after tumor cell inoculation was assessed by measuring Rluc activity by BLI. Tumor-bearing mice received $1 \times 10^{6}$ MSCs in PBS via IV injection, and then the migration of the transduced MSCs was tracked in vivo by BLI. We separated tumor-bearing mice into two groups, namely, MSC/Fluc2 and MSC-CXCR4/Fluc2. Each group consisting of three mice.

BLI was performed with an IVIS Lumina II optical imaging system (Caliper Life Sciences, Hopkinton, MA). For Fluc activity, five minutes prior to imaging, each mouse was given a $100 \mu \mathrm{l}$ of D-luciferin ( $150 \mathrm{mg} / \mathrm{kg}$ body weight) by IP injection. The luminescent photographic images of each mouse were acquired with one-minute exposure time and medium binning. For Rluc activity, coelenterazine $20 \mu \mathrm{l}$ $(10 \mathrm{mg} / 5 \mathrm{ml}$ ethanol) was mixed with sodium phosphate buffer and injected $200 \mu \mathrm{l}$ per mouse by IV and immediately started the IVIS imaging with less than 20 seconds exposure time and medium binning for measurements up to maximum photon flux. The images were quantified separately drawn a ROI at lungs and tumor region and then measured by live image software.

2.11. Immunohistochemistry. To confirm the MSC/Fluc2 and MSC-CXCR4/Fluc2 after $24 \mathrm{~h}$ tracking, tissues fixed in $10 \%$ formalin were dehydrated and embedded in paraffin. Paraffin-embedded sections of $5 \mu \mathrm{m}$ were prepared according to standard protocols. Anti-GFP antibody (Millipore, USA) was added and stained with a DAB kit. The GFP-positive staining was visualized under a light microscope at $40 \mathrm{x}$ magnifications.

\section{Statistical Analysis}

In vitro experiments were performed in three individual measurements, and in vivo experiments with three mice data were used for analyzing the means \pm standard deviation (SD); the $P$ value $<0.05$ was considered as statistically significant by Student $t$-test. $R^{2}$ was analyzed by using the Excel sheet.

\section{Results}

4.1. Characterization of CXCR4-Transduced MSCs. Transduced MSC-CXCR4/Fluc2 cells were generated by retroviral transfection, and eGFP-positive cells were sorted by FACS Aria III. The BLI signal in MSC-CXCR4/Fluc2 cells increased as the number of cells increased (Figure $1(\mathrm{a}), R^{2}=0.92$ ), and eGFP was assessed by confocal microscopy (Figure 1(b)). We also examined eGFP expression by flow cytometry (Additional Figure 2). CXCR4 mRNA and protein expression were confirmed by RT-PCR and Western blotting, respectively (Figures 1 (c) and 1(d)).

4.2. Characterization of MSC/Fluc2. The eGFP- and Fluc2-transduced MSC/Fluc2 cell lines were created by retroviral transfection, and then eGFP-positive cells were sorted by FACS Aria III. The BLI signal in MSC/Fluc2 increased as the number of cells increased (Figure 2(a), 

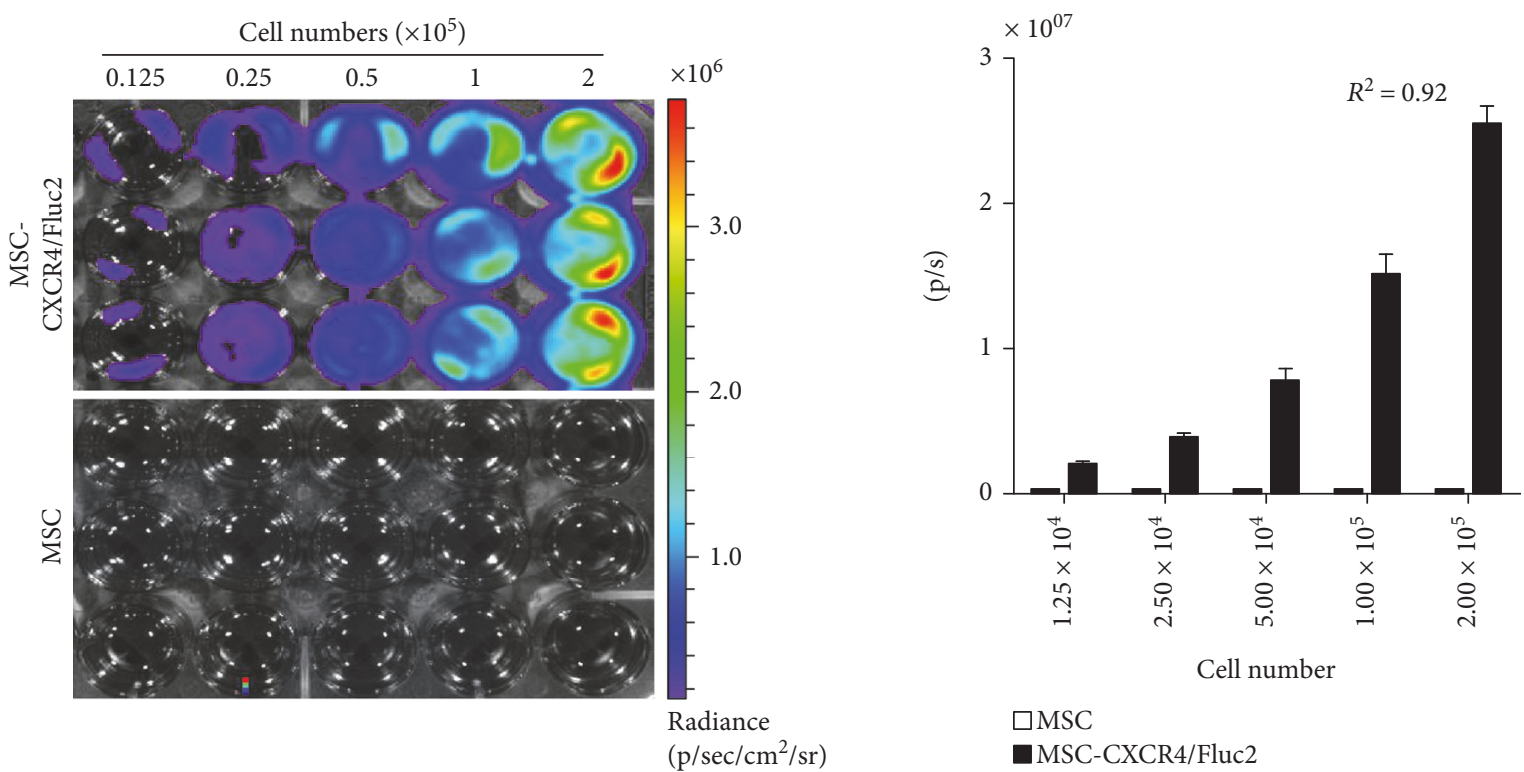

(a)
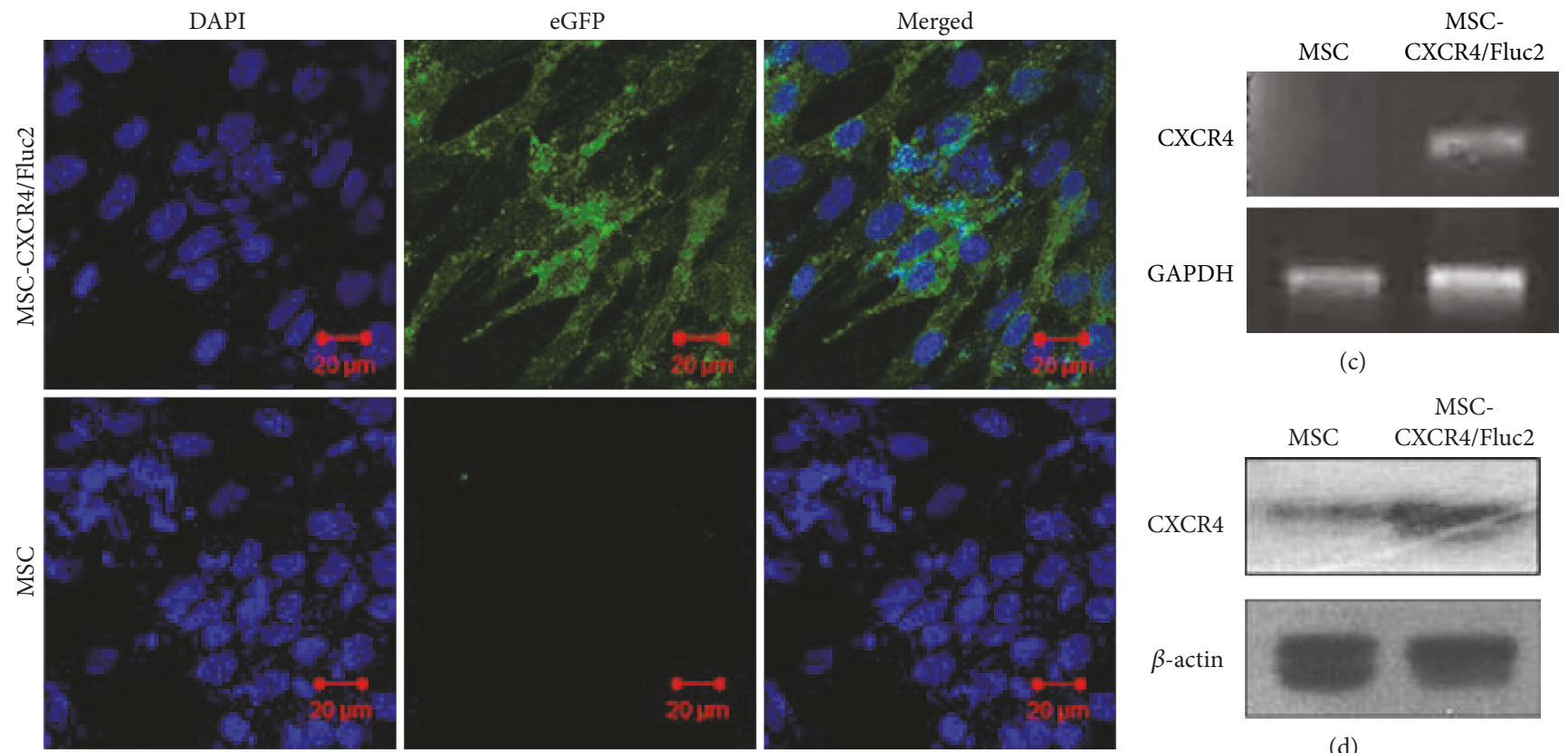

(c)

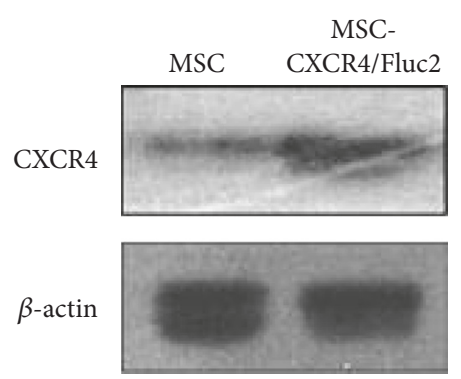

(b)

(d)

FIgure 1: Transduction of CXCR4 in mesenchymal stromal cells (MSCs) with reporter genes. (a) Fluc activity and quantitative bioluminescent imaging (BLI) data of CXCR4-transduced MSCs with different cell numbers. (b) Enhanced green fluorescent protein (eGFP) expression analysis by confocal microscopy imaging and (c) CXCR4 mRNA expression analysis by RT-PCR. (d) Protein expression of CXCR4 by Western blot analysis.

$\left.R^{2}=0.91\right)$; eGFP expression was shown by fluorescent microscopy (Figure 2(b)).

4.3. Characterization of MDAMB231/Rluc Cells. The Rluc activities of stably transduced MDAMB231/Rluc and parental MDAMB231 cells were determined by BLI. The bioluminescence activity of MDAMB231/Rluc cells increased as the number of cells increased (Figure $3(\mathrm{a}) ; R^{2}=0.92$ ). mCherry was confirmed by fluorescent microscopy (Figure 3(b)). Rluc mRNA and protein expression were confirmed by RT-PCR and Western blotting, respectively (Figures 3(c) and 3(d)).

4.4. In Vitro Migration of Transduced MSCs. In an in vitro study, bone marrow-derived MSCs containing CXCR4 (MSC-CXCR4/Fluc2) showed more migration into the lower compartment (containing $0.5 \%$ FBS) than naive MSCs in a chamber assay (Figure 4) after 6 hours. 


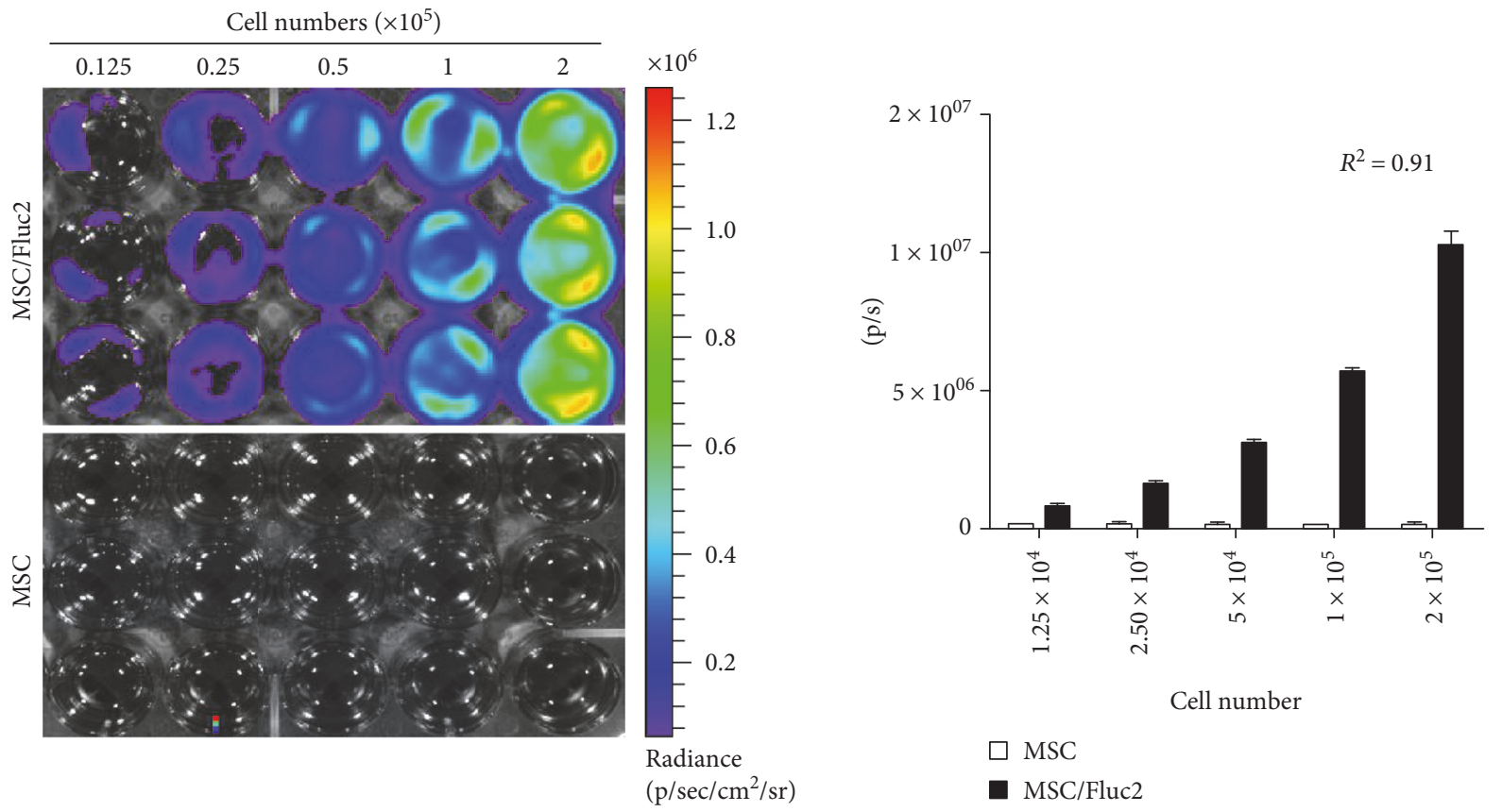

(a)
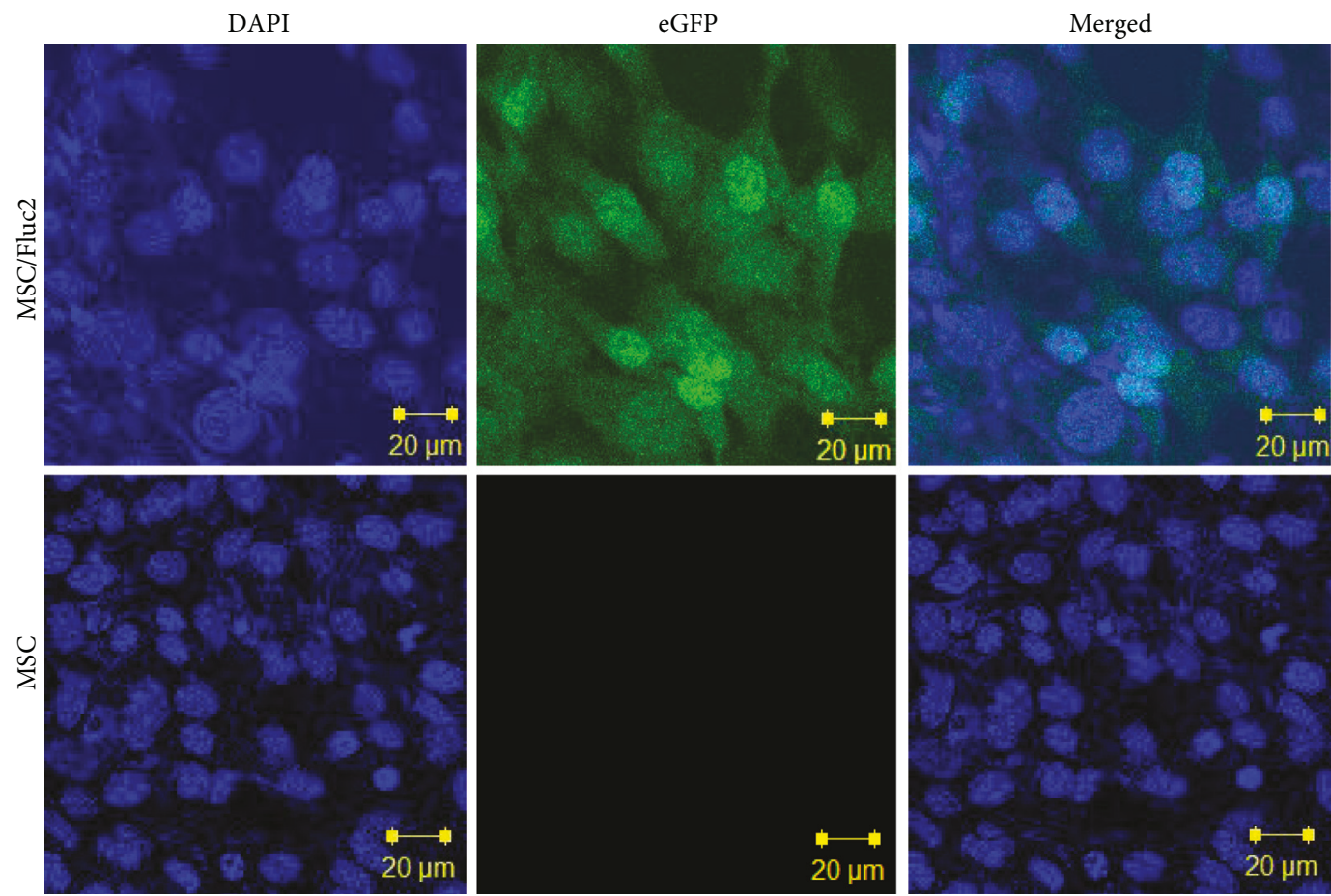

(b)

FIgure 2: Characterization of MSC/Fluc2 cells (a) BLI and quantitation of Fluc activity in transduced MSCs (MSC/Fluc2) at various concentrations. (b) eGFP confocal microscopy in transduced MSCs (MSC/Fluc2).

4.5. In Vivo Migration of MSCs. To monitor the in vivo migration of systemically transduced MSCs, we performed BLI to track the MSCs. In this study, we confirmed that the migration of MSCs toward breast cancer cells after intravenous delivery could be visualized by in vivo cell tracking using BLI. We used noninvasive systemic delivery and in vivo imaging to examine the tumor-targeting property of transduced MSCs. MSC/Fluc2 and MSC-CXCR4/Fluc2 were injected $\left(1 \times 10^{6}\right)$ into tumor-bearing mice through the tail vein, and MSC localization was assessed by BLI at 1 and 24 hours after injection (Figure 5(b)). The imaging showed that at 1 and $24 \mathrm{~h}$ after tail vein injection, the MSCs moved from the lung to the tumor sites. We quantified the lung and tumor region Fluc activity by drawn ROI separately at 


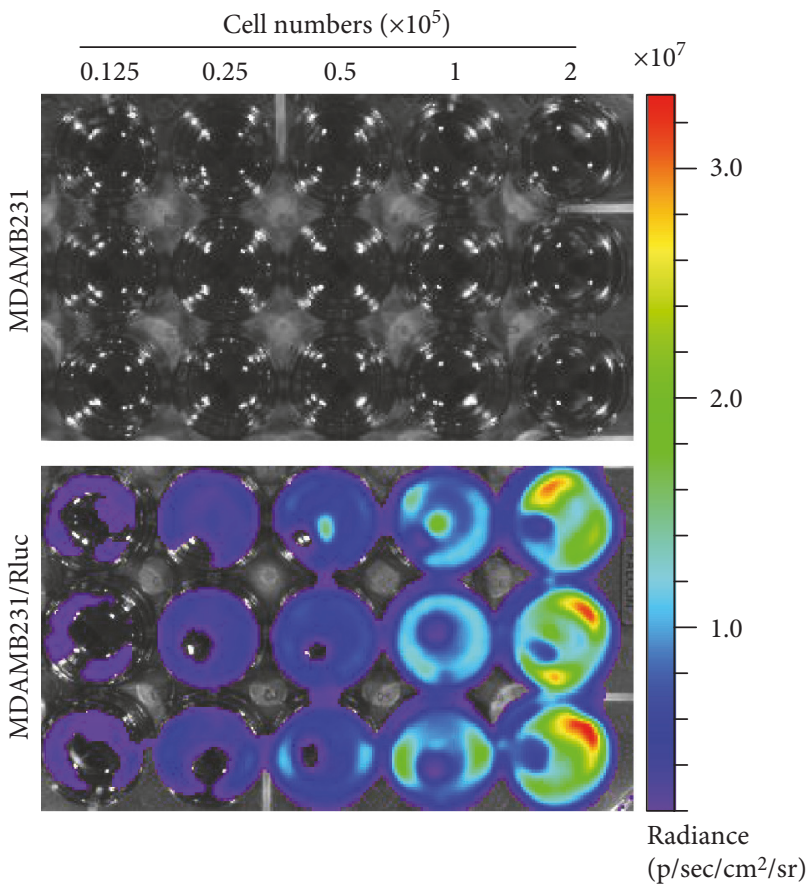

(a)
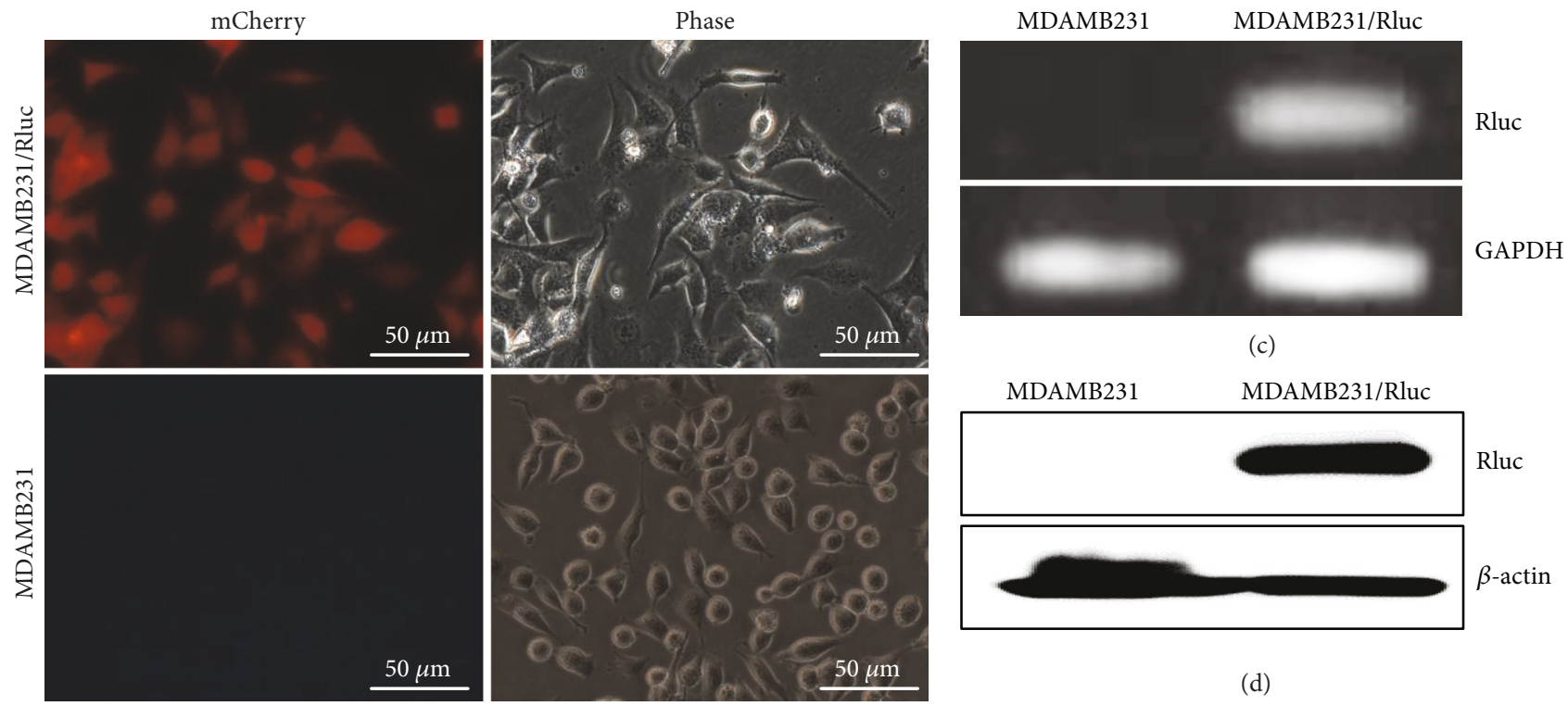

(c)

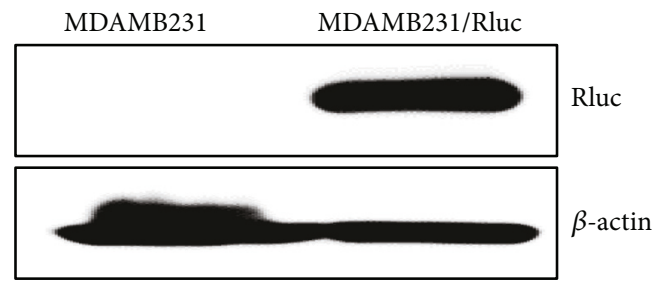

(d)

(b)

FIgURE 3: Characterization of MDAMB231/Rluc. (a) Rluc activity assessed by bioluminescent imaging (BLI) and quantitative analysis of MDAMB231/Rluc at different concentrations. (b) Transduced MDAMB231/Rluc cells were strongly positive for mCherry by fluorescence microscopy. (c) Rluc mRNA expression by RT-PCR. (d) Rluc protein expression by Western blotting.

both regions and found that Fluc activity was decreased at $24 \mathrm{~h}$ (Figure 5(c)) in the lungs. More MSC-CXCR4/Fluc2 migrated to tumor sites than MSC/Fluc2 at both 1 and 24 hours after tail vein injection (Figure 5(d)). We also confirmed higher Fluc activity of the MSCs in the tumors by ex vivo tumor imaging (Figure 5(e)). Furthermore, MSC/Fluc2 and MSC-CXCR4/Fluc2 cells after $24 \mathrm{~h}$ injection in tumor regions, we performed IHC for GFP-positive cells. IHC results showed that the positive staining of MSCCXCR4/Fluc2 was higher, when compared, than MSC/Fluc2
(Figure 5(f)). The Rluc activity of MDAMB23/Rluc was shown in a xenograft mice model (Figure 5(a)), and the in vivo results showed that the tumor recruits more CXCR4-overexpressing MSCs than native MSCs.

\section{Discussion}

For our study, MSCs were labeled with luciferase by retroviral transduction, and in vitro and in vivo migration studies demonstrated that engineered MSCs showed enhanced 

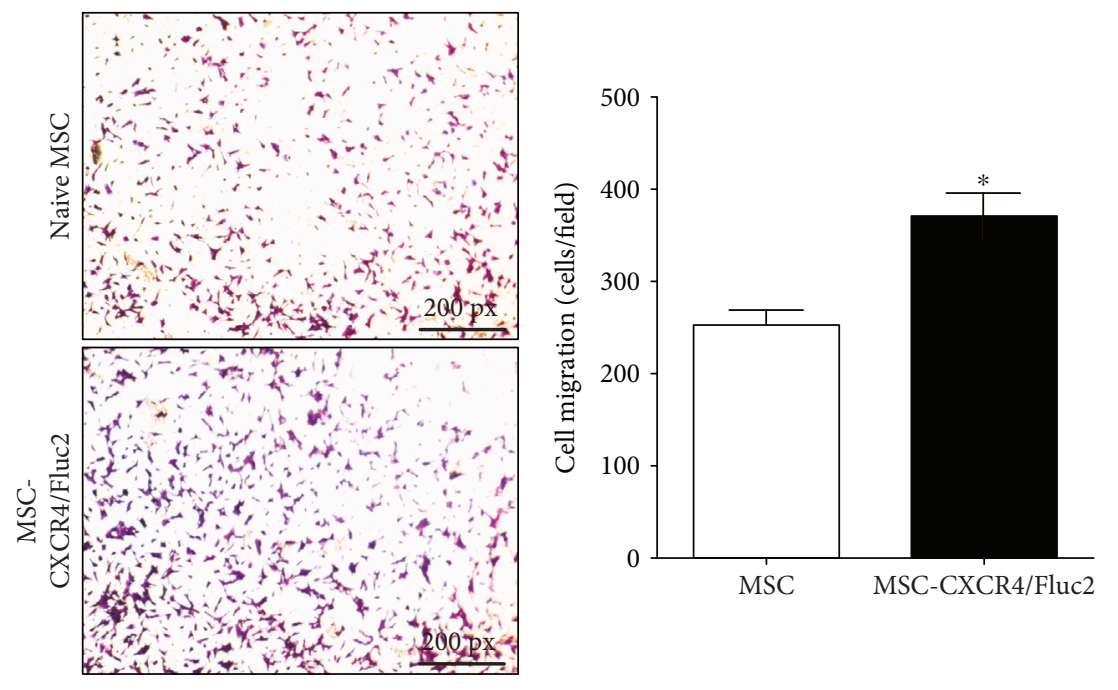

Figure 4: Chemotaxis assay for MSC-CXCR4/Fluc2. MSCs and MSC-CXCR4/Fluc2 in serum-free medium were placed into the upper well of a 24-well Transwell plate, and $0.5 \%$ FBS was added to the lower well. The plates were incubated for $6 \mathrm{~h}$, and then cells that migrated into the lower well were fixed, stained with crystal violet, and photographed in $4 \mathrm{x}$ magnification. One representative experiment of three is shown.

migratory ability towards tumors. These Fluc2-labeled MSCs could be used for noninvasive in vivo visualization and have potential for use in preclinical protocols. The increasing clinical use of MSCs in cellular and gene therapies for various diseases, including genetic and malignant disorders, is limited by the low and/or transient engraftment of transplanted MSCs [29]. The main source of MSCs, bone marrow, was taken into consideration for clinical use. The transplanted MSCs were engineered to contain therapeutic genes that display tropism for the tumors, and MSCs have been shown to exert significant antitumor effects in a human glioma xenograft mouse model [30-32]. The progenitor cells express CXCR4, which helps target the cells to injury sites when bound to SDF-1. Cultured bone marrow-derived MSCs are strongly attracted to SDF-1 due to the expression of CXCR4 [33]. However, during expansion of the MSCs in ex vivo culture, CXCR4 expression is downregulated; thus, the MSCs lose their migration potential [21]. Therefore, CXCR4 overexpression was used to increase the homing of systemically delivered MSCs towards infarcted brain. The overexpression of CXCR4 mediated the migration of MSCs towards an SDF-1 gradient in an in vitro migration assay, and systemically infused CXCR4-engineered MSCs showed strong migration towards infarcted brain when compared to naive MSCs. The CXCR4 modification did not influence the proliferation or phenotype of MSCs [34]. Genetically modified MSCs expressing CXCR4 have a typical fibroblast-like structure, similar to that of naive MSCs, and their viability and proliferation were not changed after 6 days of culture [10]. Here, we successfully transduced MSCs with CXCR4 and reporter genes (eGFP and Fluc2), and we confirmed Fluc2 activity by BLI and CXCR4 mRNA and protein expression (Figure 1). We also confirmed the in vitro migration of MSCs and MSC-CXCR4/Fluc2, as 0.5\% FBSdependent migration in a Transwell migration assay, which demonstrated the chemotactic activity of MSC-CXCR4/ Fluc2 (Figure 4). In the current study, optical molecular imaging revealed that CXCR4-overexpressing MSCs migrated to the MDAMB231/Rluc xenografts. CXCR4overexpressing MSCs could be useful for SDF1 $\alpha$-secreting breast cancer therapeutic drug delivery.

MSCs express numerous receptors and cell adhesion molecules that are involved in migration and homing to certain diseased tissues, and a significant portion are dependent on CXCR4-mediated homing $[35,36]$. The gene expression profiles of MSCs exposed to various tumor cell-derived conditioned mediums revealed the downregulation of matrix metalloproteinase- 2 and the upregulation of CXCR4. This suggests that CXCR4 and MMP-2 are involved in the multistep migration of MSC [33]. However, in previous studies, the migration of labeled MSCs was confirmed in tissue sections by immunohistochemistry, fluorescent methods, or PCR, only after the animal had been sacrificed. These methods require animals to be sacrificed at multiple time points and preparation and analysis of tissues. Because of these limitations, we used noninvasive in vivo live imaging to visualize and track the cells to assess the migration and distribution of engineered MSCs over a range of time points in the same animal.

In the current study, we found that systemically injected MSCs were distributed to the tumor region in breast cancer (MDAMB231/Rluc) xenograft mice. The injected MSCs first will go the lungs, then some time later, they will migrate to various parts of the body. After 1 hour, we found that the transduced MSCs, with and without CXCR4, reached the tumor site and remained, even after 24 hours (Figure 5(b)). The decrease of MSC/Fluc cells in the lung after $24 \mathrm{~h}$ due to the migration of cells to other inner parts of the body may be the reason. The exact mechanism for MSC-CXCR4/Fluc2 long time in the lungs is unclear. The Fluc signal from the spine region was due to cells may be present in the bone marrow of the spine region. Transplanted MSC homing to bone marrow [37]. We also confirmed the Fluc2 activity of MSC/Fluc2 and MSC-CXCR4/Fluc2 by ex vivo BLI imaging 


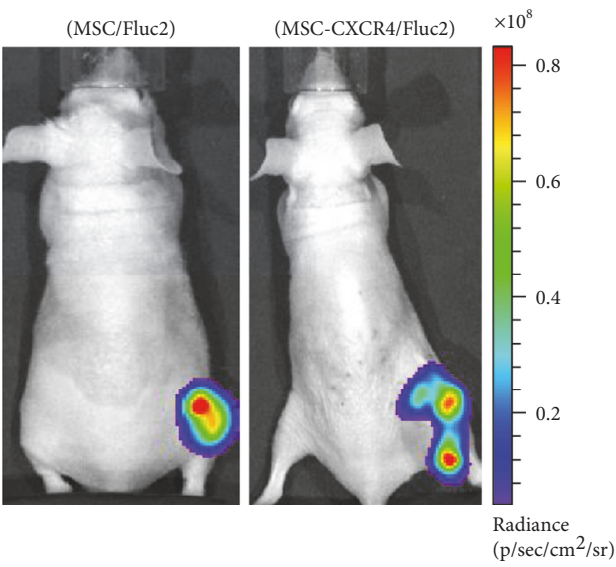

(a)
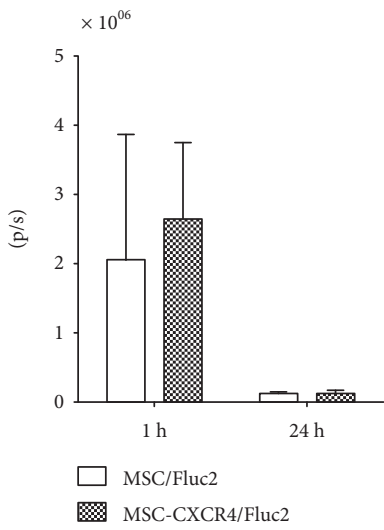

(c)

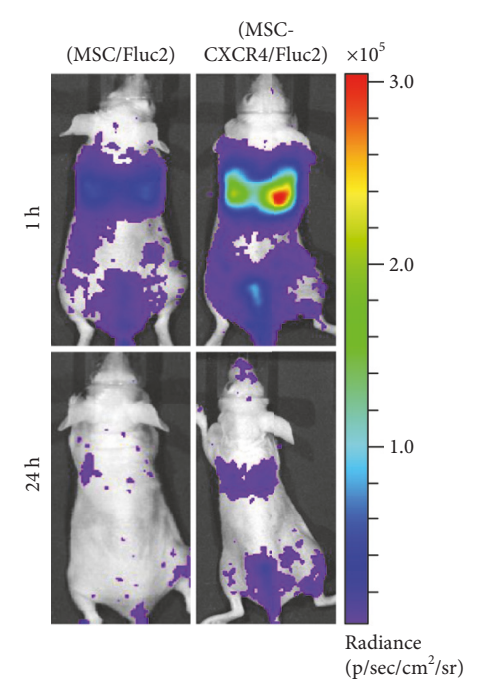

(b)

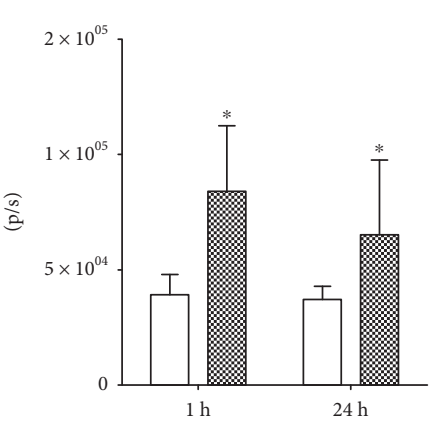

$\square$ MSC/Fluc2

冈MSC-CXCR4/Fluc2

(d)

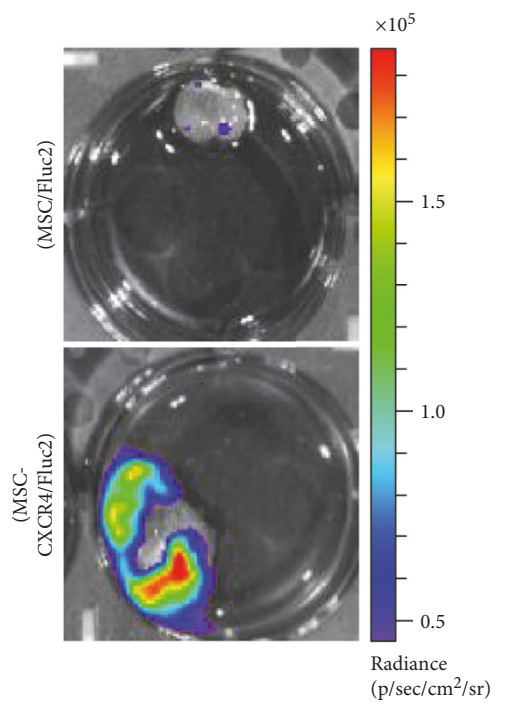

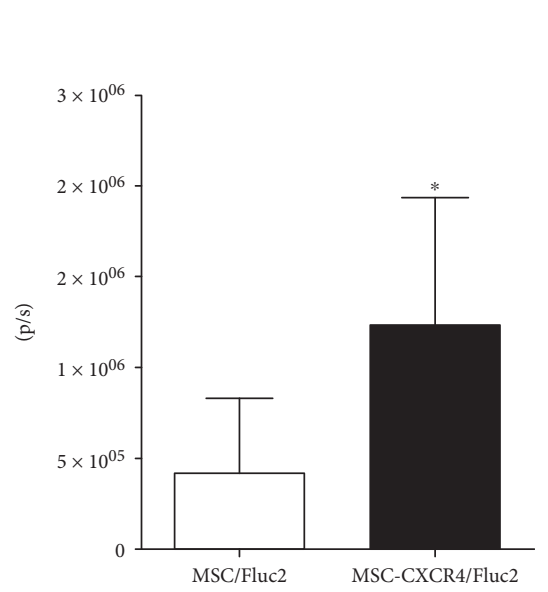

(e)

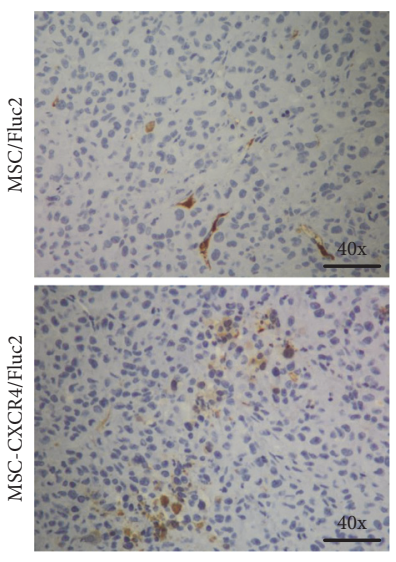

(f)

FIGURE 5: In vivo migration and localization of transplanted MSC-CXCR4/Fluc2 in the tumors. (a) Rluc activity of MDAMB231/Rluc tumor xenografts. (b) Fluc activity of migrated MSC-CXCR4/Fluc2 and MSC/Fluc2 in breast cancer xenografts. (c) Quantitative data of Fluc activity from the lungs. (d) Quantitative data of Fluc activity from tumor. (e) Ex vivo Fluc imaging of migrated MSC-CXCR4/Fluc2 and MSC/Fluc2. (f) Immunohistochemistry analysis of GFP for MSC/Fluc2 and MSC-CXCR4/Fluc cells in MDAM231/Rluc tumor. Data were analyzed from 3 mice with the means \pm standard deviation (SD), and the $P$ value $<0.05$ was considered as statistically significant by Student $t$-test. 
(Figure 5(e)), and GFP-positive cells by IHC staining showed that higher stain was obtained from MSC-CXCR4/Fluc2 injected cells (Figure 5(f)). Systemically delivered MSCs preferentially target sites of ischemic lesions, injuries, and tumors in the brain, despite their predominant entrapment in the lung vasculature [20,38]. In the present study, we found that the MSCs were transiently trapped in the lungs but then migrated over time to the tumor region, which means that MSCs administered systemically via the tail vein can reach tumors, even though some cells remain in the lungs. In addition, more CXCR4-overexpressing MSCs accumulated in the tumor than non-CXCR4-overexpressing MSCs. This might be due to the release of chemotactic factors from the tumors that attract the CXCR4-overexpressing MSCs.

\section{Conclusion}

In the current study, we showed that optical imaging can be used to reveal the distribution and homing of systemically injected MSC-CXCR4/Fluc2 and MSC/Fluc2 toward tumor sites in mice bearing breast cancer xenografts. This suggests that CXCR4-mediated cell-based therapeutics can be used to treat breast cancer. Optical imaging-based in vivo cell tracking might be a prospective imaging technique to develop MSC-mediated therapies.

\section{Conflicts of Interest}

The authors declare that they have no competing interests.

\section{Acknowledgments}

The authors thank Peter L. Hordijk (University of Amsterdam, the Netherlands) for the kind gift of plasmid CXCR4-eGFP. This research was supported by a grant from the Korea Health Technology R\&D Project, Ministry of Health \& Welfare, Republic of Korea (HI16C1501), a grant from the Medical Cluster R\&D Support Project of Daegu-Gyeongbuk Medical Innovation Foundation (DGMIF) funded by the Ministry of Health \& Welfare, Republic of Korea (HT13C0002), a grant from the Korea Health Technology R\&D Project through the Korea Health Industry Development Institute (KHIDI), funded by the Ministry of Health \& Welfare, Republic of Korea (HI15C0001), and a basic science research program grant through the NRF funded by the Ministry of Education (NRF-2016R1D1A1A02936968).

\section{References}

[1] H. L. Martin, L. Smith, and D. C. Tomlinson, "Multidrugresistant breast cancer: current perspectives," Breast Cancer: Targets and Therapy, vol. 6, pp. 1-13, 2014.

[2] K.-M. Rau, Y.-C. Lin, Y.-Y. Chen et al., "Pegylated liposomal doxorubicin (Lipo-Dox ${ }^{\circledR}$ ) combined with cyclophosphamide and 5-fluorouracil is effective and safe as salvage chemotherapy in taxane-treated metastatic breast cancer: an open-label, multi-center, non-comparative phase II study," BMC Cancer, vol. 15, no. 1, p. 1, 2015.
[3] G. Chakravarty, A. Mathur, P. Mallade et al., "Nelfinavir targets multiple drug resistance mechanisms to increase the efficacy of doxorubicin in MCF-7/Dox breast cancer cells," Biochimie, vol. 124, pp. 53-64, 2016.

[4] F. Mouh, M. Mzibri, M. Slaoui, and M. Amrani, "Recent progress in triple negative breast cancer research," Asian Pacific Journal of Cancer Prevention: APJCP, vol. 17, no. 4, pp. 1595-1608, 2015.

[5] X. Wei, X. Yang, Z.-p. Han, F.-f. Qu, L. Shao, and Y.-f. Shi, "Mesenchymal stem cells: a new trend for cell therapy," Acta Pharmacologica Sinica, vol. 34, no. 6, pp. 747-754, 2013.

[6] E.-J. Kim, N. Kim, and S.-G. Cho, "The potential use of mesenchymal stem cells in hematopoietic stem cell transplantation," Experimental \& Molecular Medicine, vol. 45, no. 1, article e2, 2013.

[7] K. Gelse, K. von der Mark, T. Aigner, J. Park, and H. Schneider, "Articular cartilage repair by gene therapy using growth factor-producing mesenchymal cells," Arthritis and Rheumatism, vol. 48, no. 2, pp. 430-441, 2003.

[8] J. Park, J. Ries, K. Gelse et al., "Bone regeneration in critical size defects by cell-mediated BMP-2 gene transfer: a comparison of adenoviral vectors and liposomes," Gene Therapy, vol. 10, no. 13, pp. 1089-1098, 2003.

[9] A. Van Damme, M. Chuah, F. Dell'Accio et al., "Bone marrow mesenchymal cells for haemophilia A gene therapy using retroviral vectors with modified long-terminal repeats," Haemophilia, vol. 9, no. 1, pp. 94-103, 2003.

[10] Z. Cheng, L. Ou, X. Zhou et al., “Targeted migration of mesenchymal stem cells modified with CXCR4 gene to infarcted myocardium improves cardiac performance," Molecular Therapy, vol. 16, no. 3, pp. 571-579, 2008.

[11] J. Tang, Q. Xie, G. Pan, J. Wang, and M. Wang, “Mesenchymal stem cells participate in angiogenesis and improve heart function in rat model of myocardial ischemia with reperfusion," European Journal of Cardio-Thoracic Surgery, vol. 30, no. 2, pp. 353-361, 2006.

[12] M. F. Pittenger, A. M. Mackay, S. C. Beck et al., "Multilineage potential of adult human mesenchymal stem cells," Science, vol. 284, no. 5411, pp. 143-147, 1999.

[13] R. Izadpanah, C. Trygg, B. Patel et al., "Biologic properties of mesenchymal stem cells derived from bone marrow and adipose tissue," Journal of Cellular Biochemistry, vol. 99, no. 5, pp. 1285-1297, 2006.

[14] P. A. Zuk, M. Zhu, P. Ashjian et al., "Human adipose tissue is a source of multipotent stem cells," Molecular Biology of the Cell, vol. 13, no. 12, pp. 4279-4295, 2002.

[15] Y. Zhang, C. Li, X. Jiang et al., "Human placenta-derived mesenchymal progenitor cells support culture expansion of long-term culture-initiating cells from cord blood CD3+ cells," Experimental Hematology, vol. 32, no. 7, pp. 657664, 2004.

[16] M. G. Roubelakis, K. I. Pappa, V. Bitsika et al., "Molecular and proteomic characterization of human mesenchymal stem cells derived from amniotic fluid: comparison to bone marrow mesenchymal stem cells," Stem Cells and Development, vol. 16, no. 6, pp. 931-952, 2007.

[17] K. Bieback, S. Kern, H. Klüter, and H. Eichler, "Critical parameters for the isolation of mesenchymal stem cells from umbilical cord blood," Stem Cells, vol. 22, no. 4, pp. 625634, 2004. 
[18] A. Erices, P. Conget, and J. J. Minguell, "Mesenchymal progenitor cells in human umbilical cord blood," British Journal of Haematology, vol. 109, no. 1, pp. 235-242, 2000.

[19] I. M. Barbash, P. Chouraqui, J. Baron et al., "Systemic delivery of bone marrow-derived mesenchymal stem cells to the infarcted myocardium feasibility, cell migration, and body distribution," Circulation, vol. 108, no. 7, pp. 863-868, 2003.

[20] R. H. Lee, A. A. Pulin, M. J. Seo et al., "Intravenous hMSCs improve myocardial infarction in mice because cells embolized in lung are activated to secrete the anti-inflammatory protein TSG-6," Cell Stem Cell, vol. 5, no. 1, pp. 54-63, 2009.

[21] R. F. Wynn, C. A. Hart, C. Corradi-Perini et al., "A small proportion of mesenchymal stem cells strongly expresses functionally active CXCR4 receptor capable of promoting migration to bone marrow," Blood, vol. 104, no. 9, pp. 2643-2645, 2004.

[22] F. Belema-Bedada, S. Uchida, A. Martire, S. Kostin, and T. Braun, "Efficient homing of multipotent adult mesenchymal stem cells depends on FROUNT-mediated clustering of CCR2," Cell Stem Cell, vol. 2, no. 6, pp. 566-575, 2008.

[23] L. A. Marquez-Curtis and A. Janowska-Wieczorek, "Enhancing the migration ability of mesenchymal stromal cells by targeting the SDF-1/CXCR4 axis," BioMed Research International, vol. 2013, Article ID 561098, 15 pages, 2013.

[24] M. Honczarenko, Y. Le, M. Swierkowski, I. Ghiran, A. M. Glodek, and L. E. Silberstein, "Human bone marrow stromal cells express a distinct set of biologically functional chemokine receptors," Stem Cells, vol. 24, no. 4, pp. 1030-1041, 2006.

[25] Y. Zhang, A. Foudi, J.-F. Geay et al., "Intracellular localization and constitutive endocytosis of CXCR4 in human CD34+ hematopoietic progenitor cells," Stem Cells, vol. 22, no. 6, pp. 1015-1029, 2004.

[26] J. Kahn, T. Byk, L. Jansson-Sjostrand et al., "Overexpression of CXCR4 on human CD34+ progenitors increases their proliferation, migration, and NOD/SCID repopulation," Blood, vol. 103, no. 8, pp. 2942-2949, 2004.

[27] J. E. Kim, S. Kalimuthu, and B.-C. Ahn, "In vivo cell tracking with bioluminescence imaging," Nuclear Medicine and Molecular Imaging, vol. 49, no. 1, pp. 3-10, 2015.

[28] P. E. de Almeida, J. R. van Rappard, and J. C. Wu, "In vivo bioluminescence for tracking cell fate and function," American Journal of Physiology-Heart and Circulatory Physiology, vol. 301, no. 3, pp. H663-H671, 2011.

[29] E. M. Horwitz, D. J. Prockop, P. L. Gordon et al., "Clinical responses to bone marrow transplantation in children with severe osteogenesis imperfecta," Blood, vol. 97, no. 5, pp. 1227-1231, 2001.

[30] S. M. Kim, C. H. Jeong, J. S. Woo, C. H. Ryu, J.-H. Lee, and S.-S. Jeun, "In vivo near-infrared imaging for the tracking of systemically delivered mesenchymal stem cells: tropism for brain tumors and biodistribution," International Journal of Nanomedicine, vol. 11, pp. 13-23, 2016.

[31] H. Miletic, Y. Fischer, S. Litwak et al., "Bystander killing of malignant glioma by bone marrow-derived tumor-infiltrating progenitor cells expressing a suicide gene," Molecular Therapy, vol. 15, no. 7, pp. 1373-1381, 2007.

[32] S. M. Kim, J. H. Oh, S. A. Park et al., "Irradiation enhances the tumor tropism and therapeutic potential of tumor necrosis factor-related apoptosis-inducing ligand-secreting human umbilical cord blood-derived mesenchymal stem cells in glioma therapy," Stem Cells, vol. 28, no. 12, pp. 2217-2228, 2010.
[33] B. R. Son, L. A. Marquez-Curtis, M. Kucia et al., "Migration of bone marrow and cord blood mesenchymal stem cells in vitro is regulated by stromal-derived factor-1-CXCR4 and hepatocyte growth factor-c-met axes and involves matrix metalloproteinases," Stem Cells, vol. 24, no. 5, pp. 1254-1264, 2006.

[34] X. Yu, D. Chen, Y. Zhang et al., "Overexpression of CXCR4 in mesenchymal stem cells promotes migration, neuroprotection and angiogenesis in a rat model of stroke," Journal of the Neurological Sciences, vol. 316, no. 1, pp. 141-149, 2012.

[35] D. Zhang, G.-C. Fan, X. Zhou et al., "Over-expression of CXCR4 on mesenchymal stem cells augments myoangiogenesis in the infarcted myocardium," Journal of Molecular and Cellular Cardiology, vol. 44, no. 2, pp. 281-292, 2008.

[36] C. H. Ryu, S. A. Park, S. M. Kim et al., "Migration of human umbilical cord blood mesenchymal stem cells mediated by stromal cell-derived factor-1/CXCR4 axis via Akt, ERK, and p38 signal transduction pathways," Biochemical and Biophysical Research Communications, vol. 398, no. 1, pp. 105-110, 2010.

[37] J. Leibacher and R. Henschler, "Biodistribution, migration and homing of systemically applied mesenchymal stem/stromal cells," Stem Cell Research \& Therapy, vol. 7, no. 1, p. 7, 2016.

[38] L. Zhang, Y. Li, M. Romanko et al., "Different routes of administration of human umbilical tissue-derived cells improve functional recovery in the rat after focal cerebral ischemia," Brain Research, vol. 1489, pp. 104-112, 2012. 

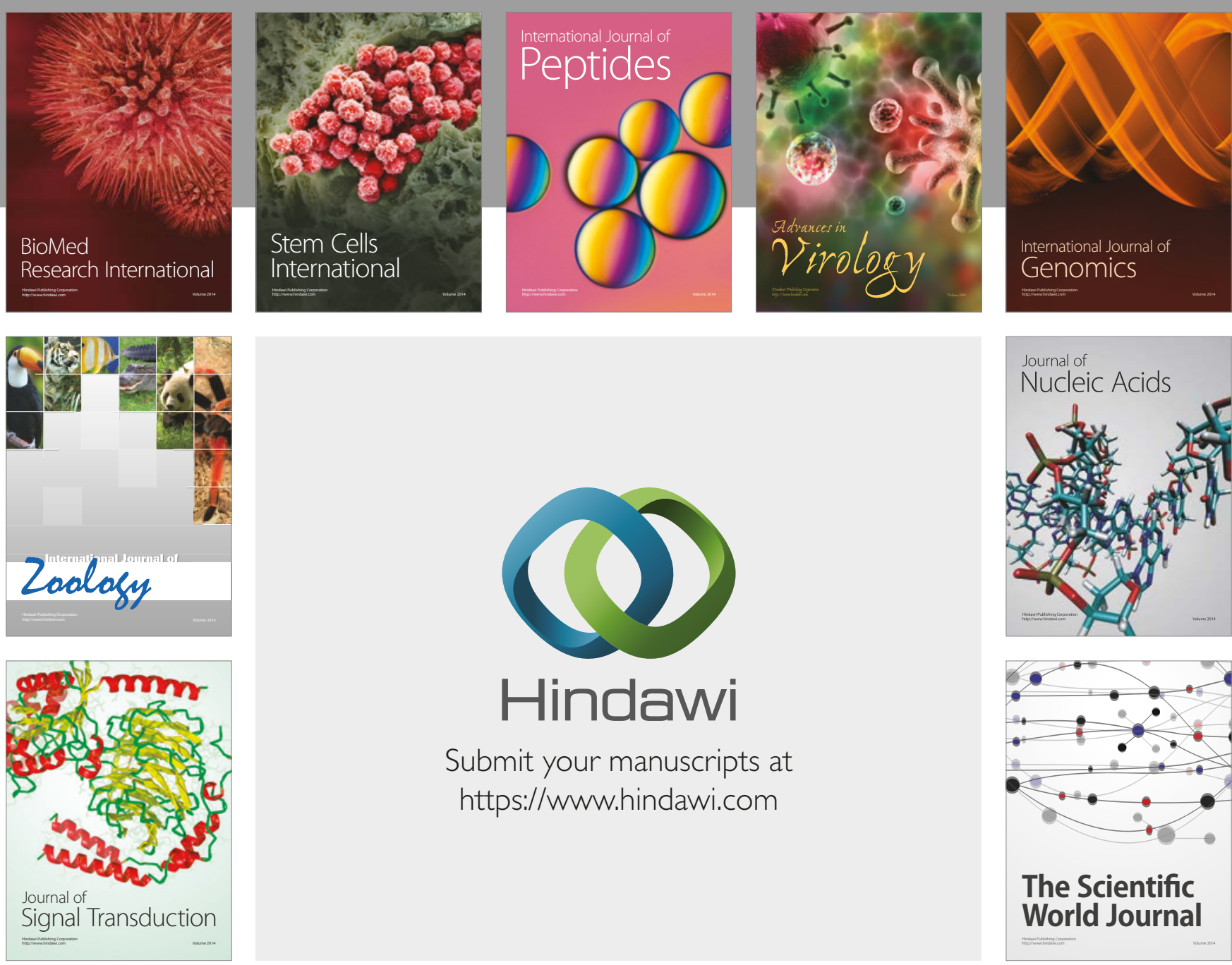

Submit your manuscripts at

https://www.hindawi.com
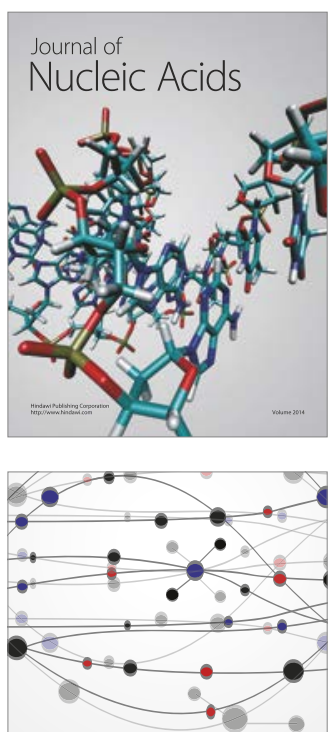

The Scientific World Journal

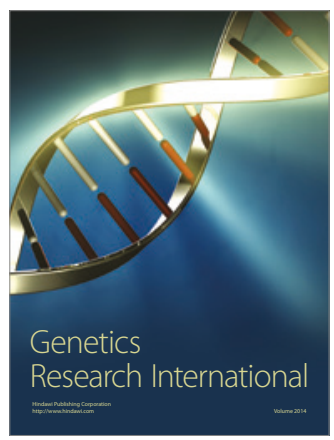

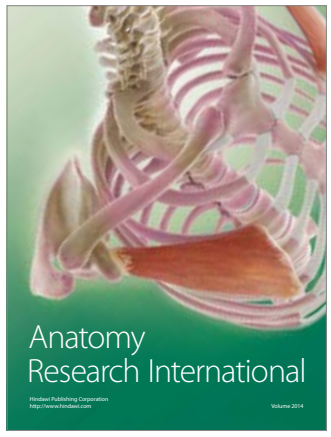

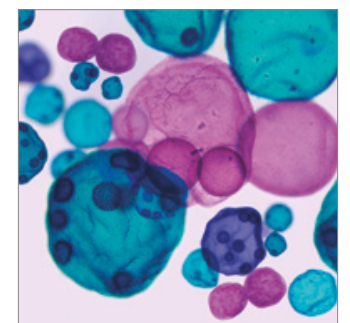

International Journal of Microbiology
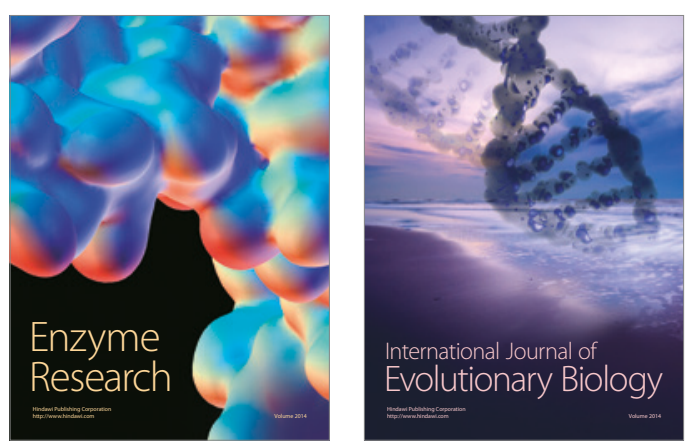
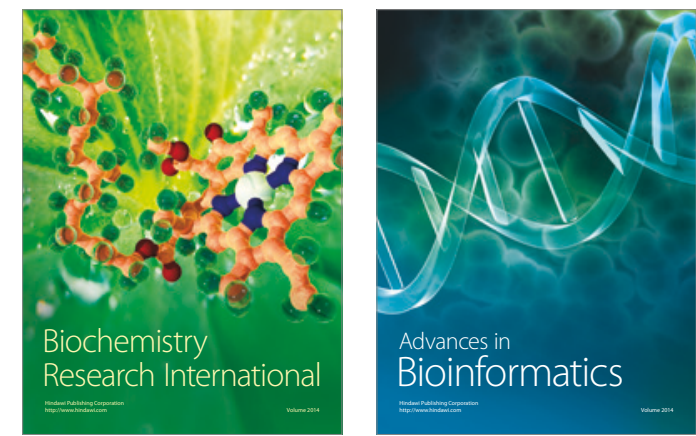

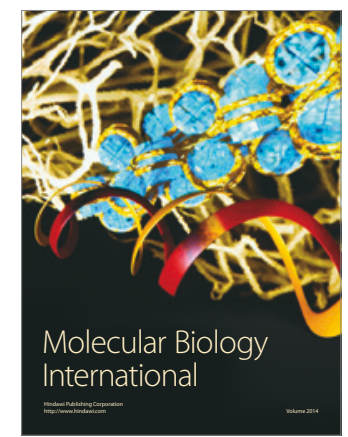

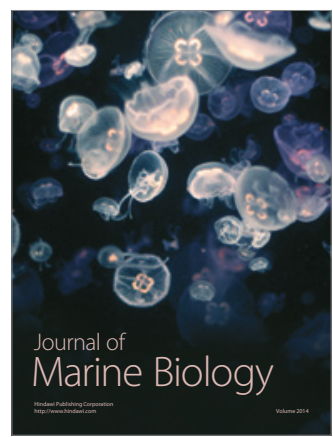

